\title{
Factors Affecting Distant Disease-Free Survival for Primary Invasive Breast Cancer: Use of a Log-Normal Survival Model
}

\author{
David R. McCready, MD; Judy-Anne W. Chapman, PhD; Wedad M. Hanna, MD; \\ Harriette J. Kahn, MD; David Murray, MD; Edward B. Fish, MD; Maureen E. Trudeau, MD; \\ Irene L. Andrulis, PhD; and H. Lavina A. Lickley, PhD
}

\begin{abstract}
Background: Invasive breast cancer is a frequently diagnosed disease that now comes with an ever expanding array of therapeutic management options. We assessed the effects of 20 prognostic factors in a multivariate context.

Methods: We accrued clinical data for 156 consecutive patients with stage 1-3 primary invasive breast cancer who were diagnosed in 1989-1990 at the Henrietta Banting Breast Center, and followed to 1995 . There is complete follow-up for $91 \%$ of patients (median follow-up of 4.9 years). The event of interest was distant recurrence (for distant disease-free survival, DFS). We used Cox and log-normal step-wise regression to assess the multivariate effects of the following factors on DFS: age, tumor size, nodal status, histology, tumor and nuclear grade, lymphovascular and perineural invasion (LVPI), ductal carcinoma-in-situ (DCIS) type, DCIS extent, DCIS at edge of tumor, ER and PgR, ERICA, adjuvant systemic therapy, ki67, S-phase, DNA index, neu oncogene, and $\mathrm{pRb}$.

Results: There was strong evidence against the Cox assumption of proportional hazards for nodal status, and nodal status was not in the Cox step-wise model. With step-wise log-normal regression, a large tumor size $(P<.001)$, positive nodes $(P=.002)$, high nuclear grade $(P=.01)$, presence of LVPI $(P=.03)$, and infiltrating duct carcinoma not otherwise specified $(P=.05)$ were associated with a reduction in DFS.

Conclusions: For nodal status, there was strong evidence against the Cox assumption of proportional hazards, and it was not included in the Cox model although it was in the log-normal model. Only traditional factors were included in the step-wise models. Thus, this statistical management of prognostic markers in breast cancer appears to be very important.
\end{abstract}

Key Words: Breast cancer-Disease-free survival-Prognostic factors.

Invasive breast cancer is a frequently diagnosed disease that now comes with an ever expanding array of therapeutic management options in the modalities of

Received September 24, 1999; accepted February 21, 2000

From the Department of Surgical Oncology (DRM), Princess Margaret Hospital, Toronto, Ontario, Canada; Henrietta Banting Breast Centre (JAWC, EBF, MET, HLAL) and Department of Pathology (WMH, HJK), Sunnybrook and Women's College Health Sciences Centre, University of Toronto, Toronto, Ontario, Canada; Pathology Department (DM), St. Michael's Hospital, University of Toronto, Toronto, Ontario, Canada; and Samuel Lunenfeld Research Institute (ILA), Mount Sinai Hospital, University of Toronto, Toronto, Ontario, Canada.

Address correspondence to: David R. McCready, MD, Department of Surgical Oncology, Princess Margaret Hospital, 610 University Avenue, Toronto, Ontario, Canada M5G 2L7; Fax: 416-946-6590; E-mail: david.mccready@uhn.on.ca surgery, radiotherapy, and systemic therapy. Decisions for an individual patient are usually made outside of randomized clinical trials, based on the best literature evidence of likely prognosis for a patient with particular demographic, clinical, and pathological characteristics.

However, clinicians are currently faced with a plethora of prognostic factors that may be relevant to predict a patient's prognosis. There is little comprehensive data to consistently support a particular set of factors being substantially better than another. Investigative reports for new prognostic markers are frequently based on univariate analyses without considering potential confounding effects of other factors ${ }^{1,2}$. The assessment of relevance for a large number of factors in itself poses a challenge to standard statistical procedures, but there has recently been growing evidence against the 
underlying assumption of proportional hazards for the standard Cox method. ${ }^{3-6}$

The Cox assumption of proportional hazards implies that the ratios of risk of recurrence (or death) for patient subgroups defined by a given prognostic factor such as nodal status ( 0 vs. $1-3$ vs. $\geq 4$ positive nodes) are approximately constant as the time from original treatment increases; a contraindication for this assumption occurs when plots of the logarithm of the cumulative hazards (risks) of recurrence or death cross. Use of the Cox model, when there is evidence that it is inappropriate, could lead to undue emphasis on a non-optimal group of prognostic factors and impede progress in clinical research and treatment. ${ }^{3}$

In this study, we simultaneously investigated the effects of a broad group of clinical and pathological factors and illustrated the use of a model-type, the log-normal, which would be appropriate when there is evidence of non-proportional hazards. The log-normal has been consistently supported by breast cancer data. ${ }^{3,7-13}$

\section{PATIENTS AND METHODS}

\section{Patients}

The Henrietta Banting Breast Center (HBBC) is a multidisciplinary assessment and treatment center for breast diseases, which opened in January, 1977. We have accrued clinical data for 156 consecutive patients with stage 1-3 primary invasive breast cancer who were diagnosed in 1989 and 1990. The patients had no previous breast or other malignancy, except in situ cervical or non-melanoma skin cancer, and were treated by a team of teaching surgeons. The patients underwent a lumpectomy and axillary dissection (116 patients) or a modified radical mastectomy (40 patients) according to National Surgical Adjuvant Breast and Bowel Project (NSABP) protocol, as a result of the decision made by the surgeon and patient. The teaching surgeons of the HBBC practice as a team, and make every effort to provide uniform treatment decisions. Each patient received good surgical management according to accepted protocols for our institution in this time period; adjuvant systemic therapy was administered by a single medical oncologist (MET).

There was complete follow-up for $91 \%$ of the patients through 1995; the median follow-up was 4.9 years, for those who are alive. The amount of remaining ipsilateral breast tissue, and thus the risk of local recurrence, varied greatly by patient. The standard practice at our institution in this time period was to provide local management for local recurrence. Only one patient with local recurrence that preceded distant recurrence received systemic therapy (chemotherapy); the patient's distant recurrence was detected the following year. Thus, the focus of this study was the association of initial tumor characteristics and distant disease progression. The event of interest was distant recurrence, where distant recurrence is defined here as outside the ipsilateral breast and includes one patient who had a regional recurrence. The time until the event was defined as distant disease-free survival (DFS). One patient who died from another cause with no evidence of recurrent breast cancer had her DFS censored at her time of death. The 1993 Dynamic 7.0 PC version of BMDP (Statistical Solutions, Saugua, MA) was used for all analyses.

\section{Factors Assessed}

The histological and immunohistochemical assessments were made by two breast pathologists (WMH and HJK) who have worked in a clinical and research partnership for several decades; the level of concordance in numerous quality control assessments is approximately $90 \%$.

Data were obtained to assess the effects of 15 traditional and 5 newer prognostic factors. The traditional factors, with their format of assessment, were the following (in univariate; in multivariate analyses): ${ }^{1}$ age $(\leq 39,40-64, \geq 65$ years [as well as by a surrogate break for menopausal status $<50, \geq 50$ ]; years), tumor size ( $\leq 20,>20 \mathrm{mms}$; mms), nodal status $(0,1-3, \geq 4$ positive; \# positive), histology (predominantly DCIS/other invasive types, infiltrating lobular, infiltrating duct NOS), tumor and nuclear grade (each as, 1/2,3 by Fisher's grading), lymphovascular and perineural invasion (LVPI: none, perineural only, lymphovascular with/without perineural), DCIS type (none, non-comedo, comedo), DCIS extent ( $\leq 20,>20 \%$; \%), DCIS at edge of tumor (absent, present), ER and $\operatorname{PgR}$ (each as, $<10, \geq 10$ $\mathrm{fmol} / \mathrm{mg}$ protein; \# fmol/mg protein), ERICA $(0,>0 \%$ positive; \%), and adjuvant tamoxifen and adjuvant chemotherapy (each as, no, yes; adjuvant systemic [no, yes]). [The handling of systemic adjuvant therapy was given extensive consideration and preliminary work-up with an examination of recurrence by separate patient subgroups and the use of stratification by treatments. Many more patients would currently be offered some form of systemic therapy; however, these study patients received fairly uniform treatment decisions from the surgical team who practiced together. Treatment decisions did not vary greatly in the years 1989-1990, the two years of patient acquisition; the decision to simply include therapy as a set of study factors that might be interactively modeled with the rest was thought to be the best approach for these data.] 
The newer factors were ki67 ( $\leq 10,>10 \%$ positive; $\%$ positive), Verity MODFIT S-phase (low, medium, high), DNA index (diploid, aneuploid determined with a cutpoint at 1.0; actual value), neu oncogene $(<2, \geq 2$ copies; number of copies), and Pharmagen $\mathrm{pRb}(<10, \geq 10 \%$ positive; $\%$ positive).

\section{Univariate Assessment}

The factor subgroups indicated above were used in the univariate assessments. Kaplan-Meier plots were made for each of the prognostic factors, and the Wilcoxon (Peto-Prentice) test statistic was used to assess the effects of the factor on DFS.

The Cox model assumes log-linear effects of the covariates on the hazard function, or risk of recurrence or death. Plots of the logarithm of the cumulative hazards for distant recurrence were plotted for each prognostic factor, with control for other factors at their means; the differences between subgroups should remain approximately constant under the Cox assumption of proportional hazards.

When there is strong evidence against proportional hazards, the class of accelerated failure time models, ${ }^{14}$ which includes the log-normal, may be a better choice; however, it then becomes necessary to select a model that is appropriate for the hazard function. For more than five decades, the log-normal distribution has been shown to be a good choice for large cohorts of breast cancer patients ${ }^{7-13}$ and stage subgroups. ${ }^{13}$ Veronesi's ${ }^{15}$ plots of annual conditional probability of distant recurrence indicate the classic shape ${ }^{14}$ for the log-normal hazard function. The log-normal survival analysis model assumes that the log of the survival time is a linear function of the prognostic factors and has approximately a normal distribution. ${ }^{14}$ From the point of view of the hazard function, the risk of an event increases from 0 to a maximum at some point in time, and decreases toward 0 thereafter. This assumption is clinically reasonable for breast cancer in that the risk of recurrence or death is expected to decrease after $\sim 3,5$, or 10 years, depending on the characteristics of the breast cancer patients. The BMDP program $2 \mathrm{~L}$ handles censored survival times and provides plots of Cox-Snell and standardized residuals to check the adequacy of the model fit. With a larger sample size and longer follow-up (more events), it would also be reasonable to confirm the log-normal distribution for events with Q-Q plots against the standard normal distribution; we have performed such analyses with other cohorts. ${ }^{16-18}$

\section{Multivariate Assessment}

We previously reported ${ }^{2,19,20}$ that it is preferable in multivariate regression analyses to fully utilize the infor- mation of continuous prognostic factors rather than to categorize such factors: i.e., tumor size in $\mathrm{cm}$, rather than the standard T1, T2, T3, or biochemical ER in fmol/mg protein, rather than as positive or negative based on a lab varying cut-point. Categorization of continuous factors may lead unjustifiably to the imputation of very different prognostic relevance for very small differences in factor values; it may also limit the multivariate investigation of factor effects ${ }^{20}$ and interactions. Thus, the factors age, tumor size, nodal status, DCIS extent, ER, PgR, ERICA, ki67, DNA index, neu oncogene, and pRb were categorized as required for univariate, but not for multivariate, investigations.

Step-wise regression was performed using the factors above in Cox and log-normal survival analyses. The model improvement for adding a factor was assessed with the likelihood ratio criterion, $-2 \log \mathrm{R}(-2 \log \mathrm{R} \sim$ $\chi^{2 *}$ under the assumption that the factor is not significantly associated with time to recurrence).

\section{RESULTS}

\section{Univariate Assessment}

Thirty-six patients experienced a distant recurrence. The number of patients by factor subgroup, number of distant recurrences, Kaplan-Meier 5-year recurrence rate, and $P$ values for tests for differences between subgroups are given in Table 1 for traditional prognostic factors, and in Table 2, for newer prognostic factors. Many of the factors exhibited significant differences between patient subgroups. Lower recurrence was observed for patients who were older $(P=.07)$, had a smaller tumor size $(P<.001)$, fewer positive nodes $(P=.004)$, tumor that was predominantly DCIS or infiltrating lobular $(P=.02)$, low/medium tumor grade $(.001)$ or nuclear grade $(P<.001)$, no LVPI $(P=.003)$, no comedo DCIS $(P=.01)$, tumor was ER positive $(P<.001)$ or $\operatorname{PgR}$ positive $(P=.005)$, received adjuvant tamoxifen $(P=.06)$ or chemotherapy $(P=.001)$, tumor had low ki67 positivity $(P=$ $.07)$, and the tumor was diploid $(P=.02)$.

Figure 1 shows the log cumulative hazard plot for nodal status after controlling for other factors; there is strong evidence against the assumption of proportional hazards in that the graphs for the patient subgroups intersect or cross. Patients who were node-positive tended to receive more adjuvant systemic therapy (Table 3). In all, $31 \%$ of nodenegative patients received systemic therapy; $89 \%$ of patients, with $1-3$ positive nodes, and $95 \%$ of patients with 4

*Categorical factors have only one format listing when they were used in the same format in both univariate and multivariate analyses. 
TABLE 1. Distant recurrence rates for traditional prognostic factors

\begin{tabular}{|c|c|c|c|c|}
\hline Factor & Patients & $\begin{array}{l}\text { No. of } \\
\text { recurrences }\end{array}$ & $\begin{array}{c}\text { Kaplan-Meier } \\
\text { 5-yr DFS } \\
(95 \% \text { CI })\end{array}$ & $P$-value ${ }^{1}$ \\
\hline \multicolumn{5}{|l|}{ Age (years) } \\
\hline$\leq 39$ & 16 & 7 & $.49(.22, .76)$ & \multirow{3}{*}{.07} \\
\hline $40-64$ & 99 & 21 & $.79(.70, .87)$ & \\
\hline$\geq 65$ & 41 & 8 & $.80(.68, .93)$ & \\
\hline \multicolumn{5}{|l|}{ Tumour size (mms) } \\
\hline$\leq 20$ & 74 & 7 & $.90(.83, .97)$ & \multirow[b]{2}{*}{$<.001$} \\
\hline$>20$ & 81 & 29 & $.64(.53, .75)$ & \\
\hline \multicolumn{5}{|l|}{ Nodal Status (No. pos.) } \\
\hline 0 & 91 & 15 & $.84(.76, .91)$ & \multirow{3}{*}{.004} \\
\hline $1-3$ & 45 & 11 & $.73(.60, .87)$ & \\
\hline$\geq 4$ & 20 & 10 & $.51(.28, .74)$ & \\
\hline \multicolumn{5}{|l|}{ Histology $^{2}$} \\
\hline Predominantly DCIS/ & 46 & 7 & $.83(.72, .95)$ & \multirow{4}{*}{.02} \\
\hline Other invasive types & & & & \\
\hline Infiltrating lobular & 18 & 1 & $1.00 \mathrm{~N} / \mathrm{A}$ & \\
\hline Infiltrating Duct NOS & 92 & 28 & $.68(.58, .78)$ & \\
\hline \multicolumn{5}{|l|}{ Tumour Grade (Fisher's) ${ }^{2}$} \\
\hline Grade $1 /$ Grade 2 & 63 & 7 & $.90(.82, .98)$ & \multirow[b]{2}{*}{.001} \\
\hline \multirow{2}{*}{\multicolumn{5}{|c|}{ Nuclear Grade (Fisher's) ${ }^{2}$}} \\
\hline & & & & \\
\hline Grade $1 /$ Grade 2 & 88 & 10 & $.89(.82, .96)$ & \multirow[b]{2}{*}{$<.001$} \\
\hline Grade 3 & 68 & 26 & $.60(.48, .72)$ & \\
\hline \multicolumn{5}{|l|}{$\mathrm{LVPI}^{2}$} \\
\hline None & 87 & 12 & $.85(.78, .93)$ & \multirow{3}{*}{.003} \\
\hline Perineural only & 15 & 4 & $.79(.57,1.00)$ & \\
\hline Lymphovascular \pm perineural & 54 & 20 & $.60(.45, .74)$ & \\
\hline \multicolumn{5}{|l|}{ DCIS Type } \\
\hline None & 70 & 14 & $.79(.68, .89)$ & \multirow{3}{*}{.01} \\
\hline Non-comedo & 63 & 11 & $.84(.74, .93)$ & \\
\hline Comedo & 23 & 11 & $.47(.25, .69)$ & \\
\hline \multicolumn{5}{|l|}{ DCIS Extent $(\%)$} \\
\hline$\leq 20$ & 141 & 32 & $.77(.69, .84)$ & \multirow[b]{2}{*}{.82} \\
\hline$>20 \%$ & 15 & 4 & $.72(.48, .96)$ & \\
\hline \multicolumn{5}{|l|}{ DCIS at Edge of Tumour } \\
\hline Absent & 99 & 18 & $.81(.73, .89)$ & \multirow[b]{2}{*}{.12} \\
\hline Present & 57 & 18 & $.68(.56, .81)$ & \\
\hline \multicolumn{5}{|l|}{ ER (fmol/mg protein) } \\
\hline$<10$ & 33 & 15 & $.51(.33, .69)$ & \\
\hline$\geq 10$ & 122 & 21 & $.83(.76, .90)$ & $<.001$ \\
\hline PgR (fmol/mg protein) & & & & \\
\hline$<10$ & 46 & 16 & $.63(.49, .78)$ & \\
\hline$\geq 10$ & 109 & 20 & $.82(.74, .89)$ & .005 \\
\hline ERICA ( $\%$ positive) & & & & \\
\hline 0 & 55 & 15 & $.71(.58, .83)$ & \\
\hline$>0$ & 101 & 21 & $.79(.71, .88)$ & .23 \\
\hline Adjuvant Tamoxifen & & & & \\
\hline No & 104 & 28 & $.72(.63, .81)$ & \\
\hline Yes & 50 & 8 & $.85(.74, .95)$ & .06 \\
\hline Adjuvant Chemotherapy & & & & \\
\hline No & 114 & 20 & $.83(.75, .90)$ & \\
\hline Yes & 40 & 16 & $.57(.41, .73)$ & .001 \\
\hline
\end{tabular}

${ }^{1}$ Based on Wilcoxon (Peto-Prentice) test statistic.

${ }^{2}$ There were 4 patients with tumors that were predominantly DCIS; 3 , with tumor grade $1 ; 2$, with nuclear grade $1 ; 3$, with lymphovascular and perineural invasion. None of the patients in these small subgroups experienced distant recurrence.

or more positive nodes. The corrected hazard rates (Fig. 1) were very similar for all three subgroups for about 15 months, after which those with $\geq 4$ positive nodes had the best experience, followed by the node-negative group and then the 1-3 positive nodes group.

\section{Multivariate Assessment}

Figure 2 shows the results of the Cox step-wise regression. There were four factors which made a significant $(P \leq .05)$ addition to the multivariate model for 
TABLE 2. Distant recurrence rates for newer prognostic factors

\begin{tabular}{|c|c|c|c|c|}
\hline Factor & Patients & $\begin{array}{l}\text { No. of } \\
\text { recurrences }\end{array}$ & $\begin{array}{c}\text { Kaplan-Meier } \\
\text { 5-yr DFS } \\
(95 \% \text { CI })\end{array}$ & $P$-value ${ }^{1}$ \\
\hline \multicolumn{5}{|l|}{ Ki67 (\% positive) } \\
\hline$\leq 10$ & 103 & 19 & $.80(.72, .88)$ & \\
\hline$>10$ & 53 & 17 & $.70(.57, .83)$ & .07 \\
\hline \multicolumn{5}{|l|}{ S-phase } \\
\hline low & 51 & 13 & $.73(.61, .86)$ & \\
\hline medium & 52 & 11 & $.79(.67, .91)$ & .98 \\
\hline high & 51 & 12 & $.77(.65, .89)$ & \\
\hline \multicolumn{5}{|l|}{ DNA index } \\
\hline diploid & 48 & 5 & $.91(.82, .99)$ & \\
\hline aneuploid & 106 & 31 & $.70(.61, .79)$ & .02 \\
\hline \multicolumn{5}{|c|}{ Neu oncogene (\# copies) } \\
\hline$<2$ & 120 & 29 & $.75(.67, .83)$ & \\
\hline$\geq 2$ & 25 & 5 & $.82(.66, .98)$ & .92 \\
\hline \multicolumn{5}{|l|}{$\mathrm{pRb}(\%$ positive $)$} \\
\hline$<10$ & 68 & 16 & $.76(.66, .87)$ & \\
\hline$\geq 10$ & 85 & 20 & $.75(.65, .85)$ & .82 \\
\hline
\end{tabular}

${ }^{1}$ Based on Wilcoxon (Peto-Prentice) test statistic.

DFS. A large tumor size $(P<.001)$, high nuclear grade $(P=.005)$, presence of LVPI $(P=.004)$, and infiltrating duct NOS $(P=.02)$ were associated with shorter DFS. The presence of DCIS at the edge of the tumor was also associated with a reduction in DFS $(P=.10)$. Nodal status was not included in the step-wise model, but there was evidence against the assumption of proportional hazards for the factor nodal status.

The results of the log-normal step-wise regression are shown in Fig. 3. A large tumor size $(P<.001)$, positive nodes $(P=.002)$, high nuclear grade $(P=.01)$, presence of LVPI $(P=.03)$, and infiltrating duct NOS $(P=.05)$ were associated with a reduction in DFS.

The model improvement with the 5 factor Cox and log-normal models was similar $(-2 \log \mathrm{R}=42.63,42.71$, respectively); however, the model improvement for the 4 factor Cox model was 39.86 .

Figures 4-8 are the Kaplan-Meier plots for DFS, respectively, for the factors tumor size, nodal status, nuclear grade, LVPI, and histology.

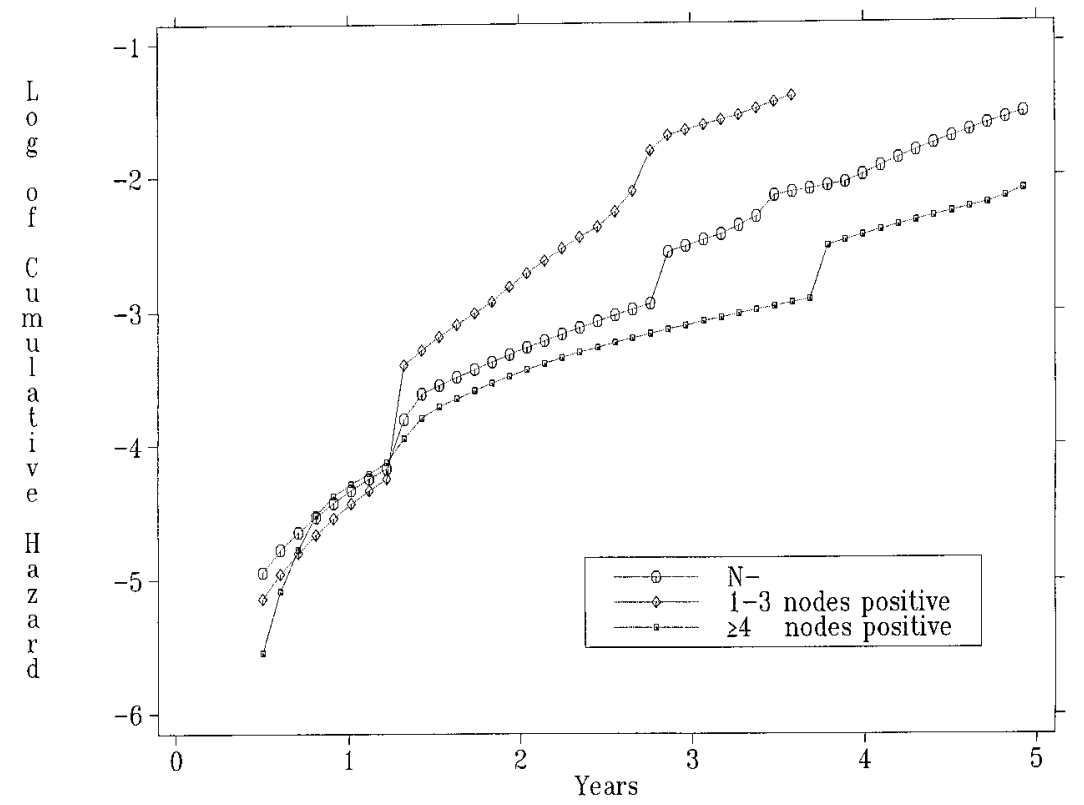

FIG. 1. Log cumulative hazard plot for distant DFS by nodal status (node-negative, $1-3$ positive, $\geqslant 4$ positive) with control for other factors at their means. 
TABLE 3. Administration of systemic therapy by nodal status

\begin{tabular}{cccccc}
\hline & & \multicolumn{3}{c}{ Nodal Status } \\
\cline { 3 - 5 } Systemic therapy & No. of Patients & Negative & $1-3$ positive & $\geq 4$ positive & $P$-value \\
\hline No & 68 & 62 & 5 & 1 & $<0.001$ \\
Yes & 86 & 28 & 39 & 19 & $<$ \\
\hline
\end{tabular}

${ }^{1}$ Based on Pearson $\chi^{2}$ test statistic.

\section{DISCUSSION}

After the diagnosis of breast carcinoma is confirmed, clinicians are faced with the decision of treatment modality: lumpectomy or mastectomy, method of assessing nodal involvement, administration (and extent of) adjuvant radiotherapy, and systemic therapy (hormonal or chemotherapy). Clinical trials data provide scientific evidence to support certain courses of action for very specific questions and subgroups of patients. The vast majority of issues that require consideration cannot be tested due to cost, time, and sometimes ethics. Clinical whole population studies, without randomization of patients or treatment decisions, provide the largest pool of evidence that must be used for medical decisions; such studies frequently involve the groups of patients for whom the next treatment decisions must be applied. However, there is a need to control the data collection and analyze such studies more carefully. ${ }^{1-4,19,21}$ In particular, it is important to consider the effects of factors in a multivariate context. ${ }^{1,2}$ It is also important to examine the appropriateness of underlying assumptions such as that of proportional hazards for the standard Cox method. ${ }^{3-6,22}$

Both Cox and log-normal ${ }^{14}$ survival analysis may be used to assess the effects of prognostic factors on the risk of recurrence or death. The tests for examining the underlying assumptions and ascertaining an appropriate choice are outlined in the methods section of this article, and are available in commercial statistical packages. We have shown in this work that the severe violation of underlying model assumptions may lead to an incorrect conclusion about whether a factor has a significant effect on recurrence or death.

Tumor size and nodal status are usually considered the two most important indicators of patient prognosis. Yet, nodal status was not included here in the Cox step-wise model, because there was strong evidence against the assumption of proportional hazards. One cannot assume

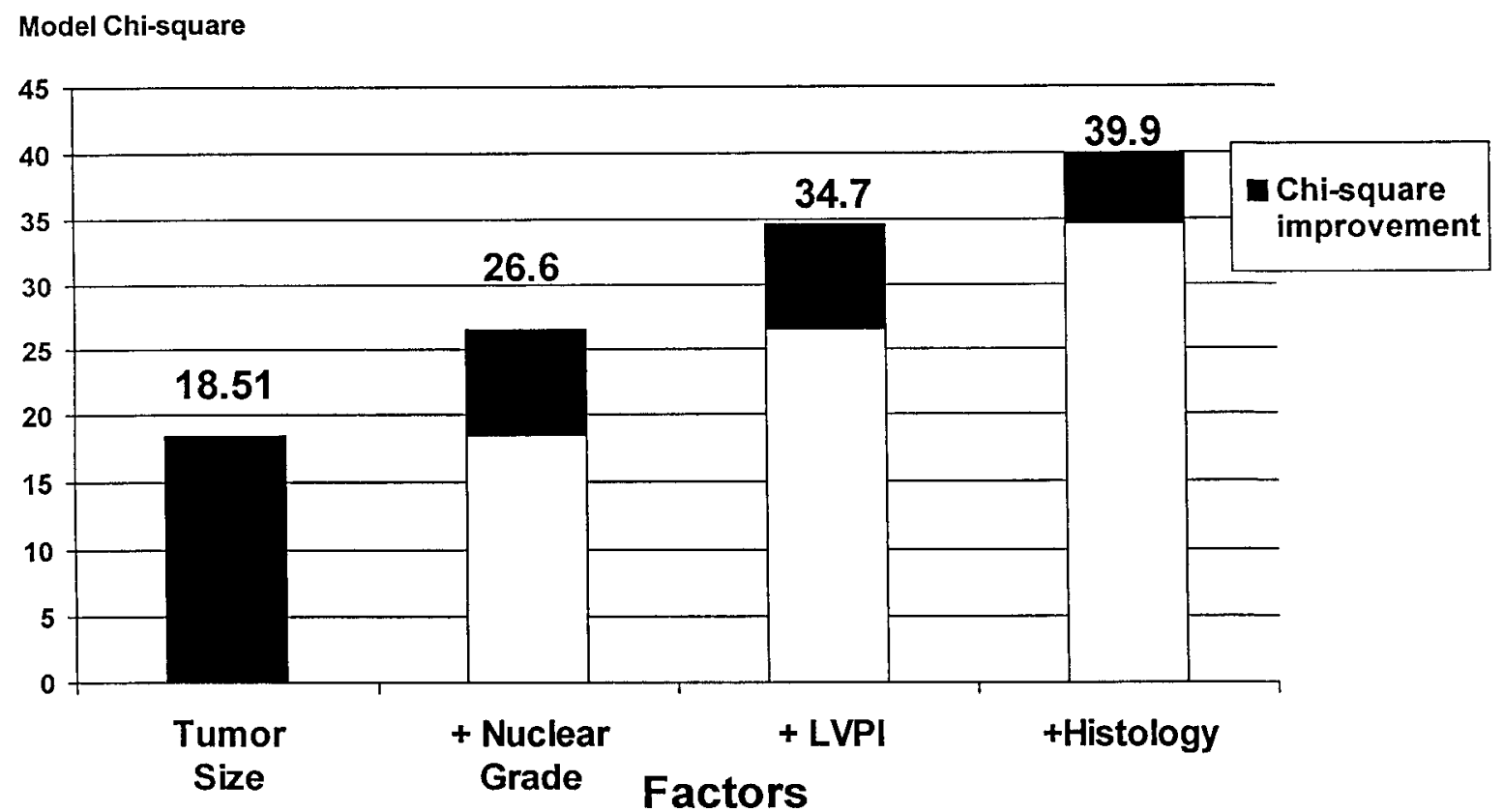

FIG. 2. Cox step-wise multivariate model building. Histograms illustrate $\chi^{2}$ improvement for multivariate model building by adding tumor size $(P<$ $.001)$, nuclear grade $(P=.005)$, LVPI $(P=.004)$, and histology $(P=.02)$. 


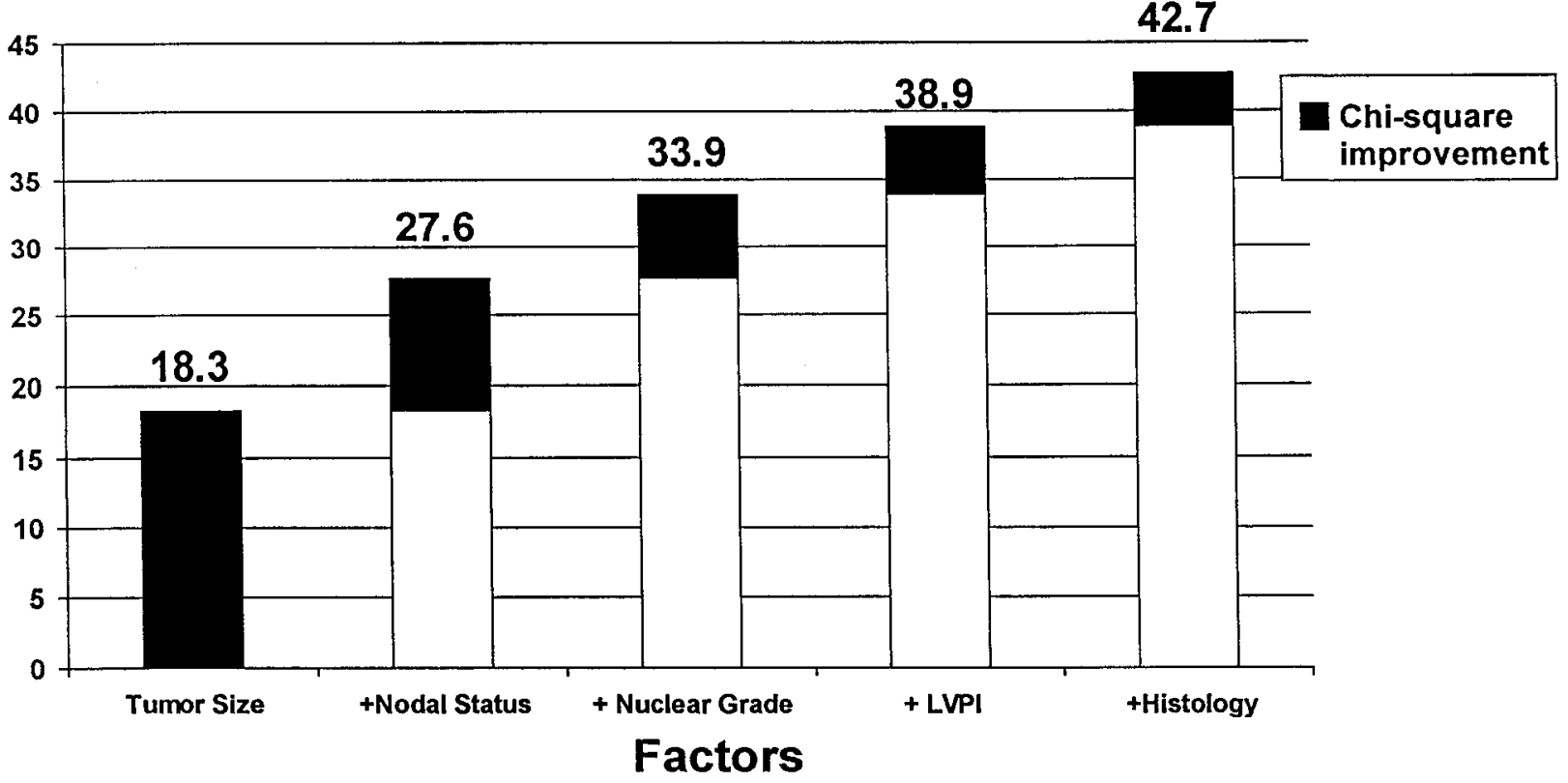

FIG. 3. Log-normal step-wise multivariate model building. Histograms illustrate $\chi^{2}$ improvement for multivariate model building by adding tumor size $(P<.001)$, nodal status $(P=.002)$, nuclear grade $(P=.01)$, LVPI $(P=.03)$, and histology $(P=.05)$.

that the Cox model is always robust enough or appropriate for statistical evaluations of breast cancer data. ${ }^{22}$ The choice of an alternate model would be dependent on the hazard (risk) pattern ${ }^{14}$ of an event (recurrence or death) for a particular cancer with the length of follow-up for the study. The log-normal model may be appropriate for primary breast cancer when there is evidence against this assumption;3,7-13 for a log-normal model, the risk is 0 at the beginning of the study, increases to a maximum, and then decreases approaching 0 with long follow-up. Breast cancer has been observed to have an increasing risk of recurrence/death which subsequently, after some point in time, decreases with many late events. ${ }^{7,8,15} \mathrm{~A}$ log-normal model has been supported in long-term investigations of breast cancer. ${ }^{3,7-13}$ Early work with the log-normal model preceded the era of high speed computers, and there were difficulties in simultaneously estimating several parameters, ${ }^{7}$ obtaining initial parameter estimates, ${ }^{7}$ differentiating between alternative model choices, ${ }^{7}$ and handling a lot of censoring. ${ }^{14}$ The availability of readily accessible statistical packages makes the routine use of a log-normal model feasible. BMDP permits a log-normal step-wise regression analysis for survival data with a full complement of residual checks.

Nodal status $(P=.002)$ was included in the step-wise log-normal model. In a previous investigation of the effects of nodal status on local recurrence, ${ }^{3}$ we had a similar situation with evidence against proportional hazards for this factor; nodal status was not included in the step-wise Cox model although it was in the step-wise log-normal. After 2 more years of follow-up, nodal status was eventually included in the step-wise Cox model. It is important to note these observations in the context that many investigators do not routinely check the appropriateness of the underlying assumptions for their statistical procedures; the magnitude of the effect that departures from assumptions might exhibit on results might not be anticipated to be this large. Certainly, most clinicians would acknowledge that they anticipate nodal status to be an important determinant of prognosis and the masking of this effect might be viewed as worrisome.

The determination of significant factors was made with the standard step-wise addition of factors, one at a time. We have been examining alternative factor selection strategies, ${ }^{2-4}$ and the results of alternative all subsets analyses will be forthcoming. The step-wise log-normal model included tumor size and lymph node status which are clinically useful ${ }^{23}$ components of pathological staging, along with histological type. Nuclear grade ${ }^{24,25}$ and $\mathrm{LVPI}^{26,27}$ are also recognized as important prognostic factors. Interestingly, this study did not find that over expression of the Her 2 neu oncogene was related to a poorer prognosis, as was found in a previous study of node-negative patients; 28 


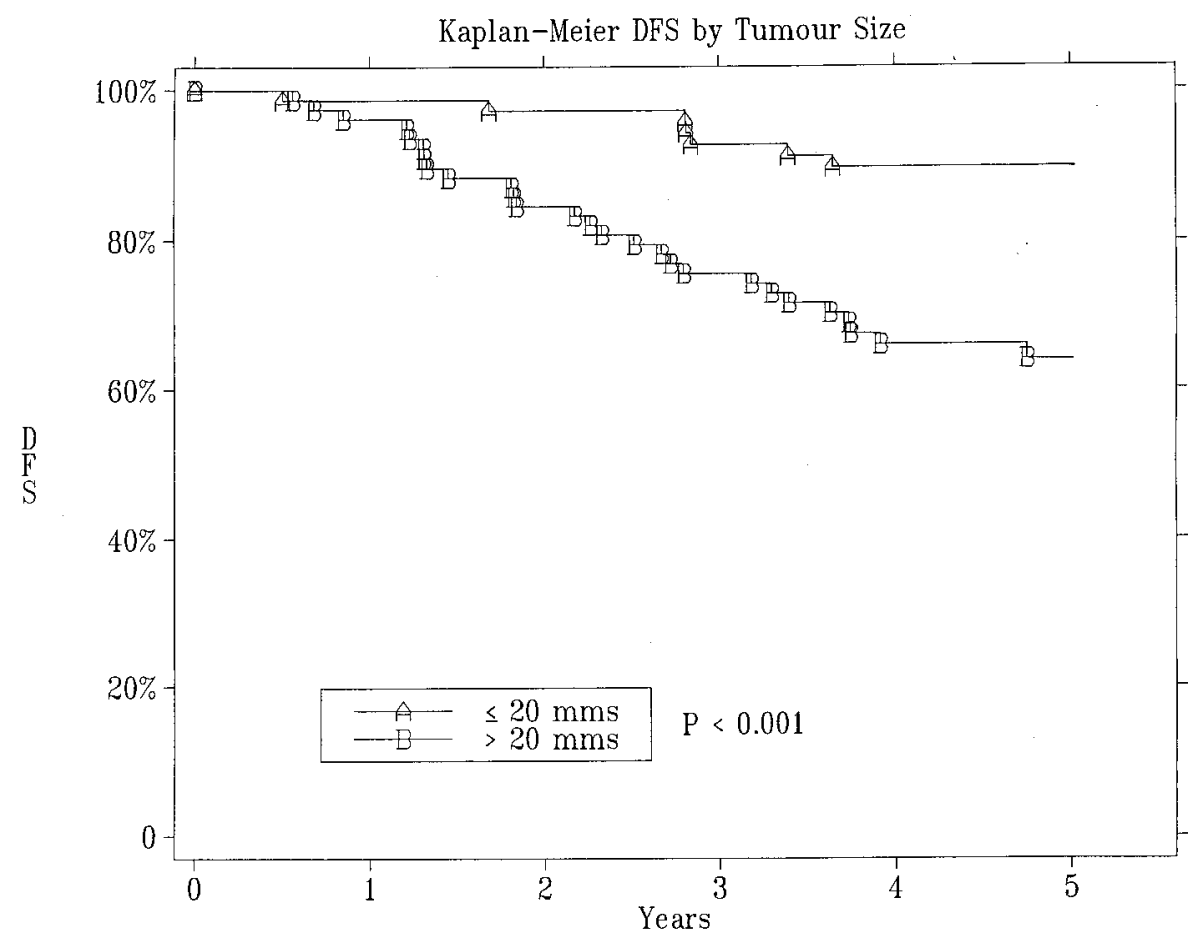

FIG. 4. Kaplan-Meier plot of distant DFS by tumor size $(\leq 20 \mathrm{mms},>20$ $\mathrm{mms}$ ).

differences between the studies include a complete standardized pathology review to include specific histological subtype, nuclear and histologic grade, and the inclusion of node positive patients in the present study. These results are for standard step-wise multivariate regression analyses.

The results reported here illustrate that standardized, well-performed traditional pathological determinants can

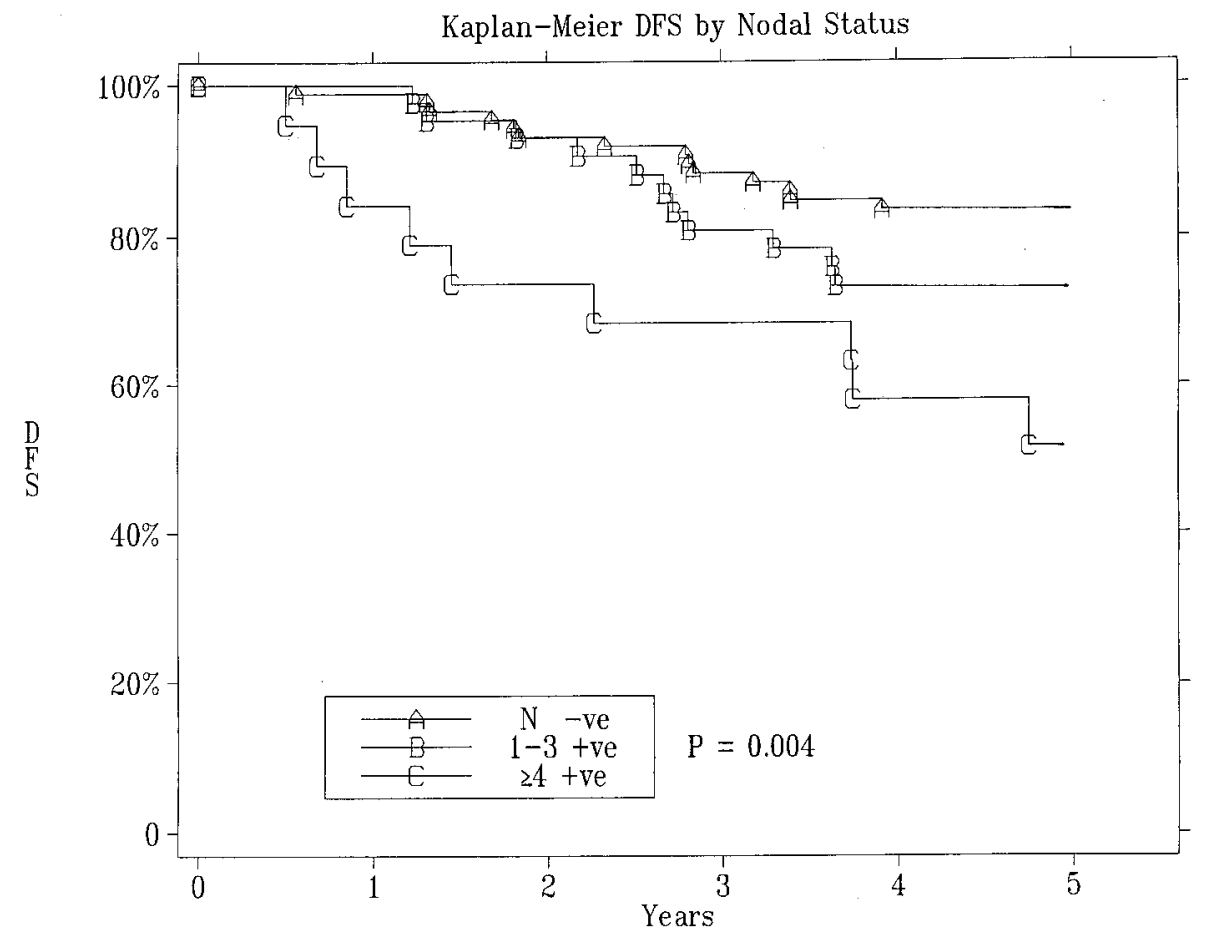

FIG. 5. Kaplan-Meier plot of distant DFS by nodal status (node-negative, 1-3 positive, $\geq 4$ positive). 


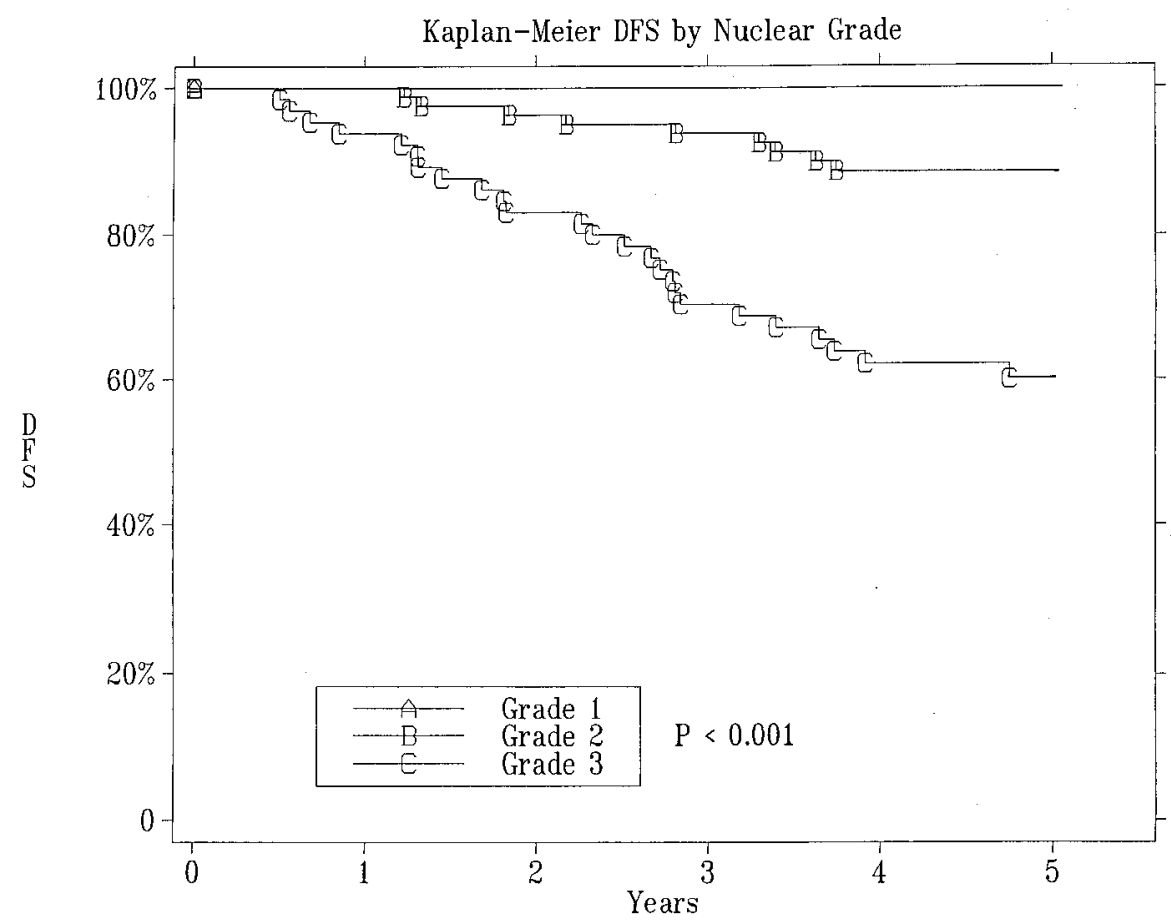

FIG. 6. Kaplan-Meier plot of distant DFS by nuclear grade (Fisher's grade $1,2,3)$.

be effective in classifying prognosis, and that some newer putative factors should be tested within a full database with all traditional factors, using appropriate statistical methods, before they are used in clinical decision making. ${ }^{23}$
Both adjuvant tamoxifen and adjuvant chemotherapy had significant univariate associations with DFS, but the combined factor adjuvant systemic therapy was not included in the step-wise model with either a Cox or log-normal model. Many clinical trials have indicated a substantial survival

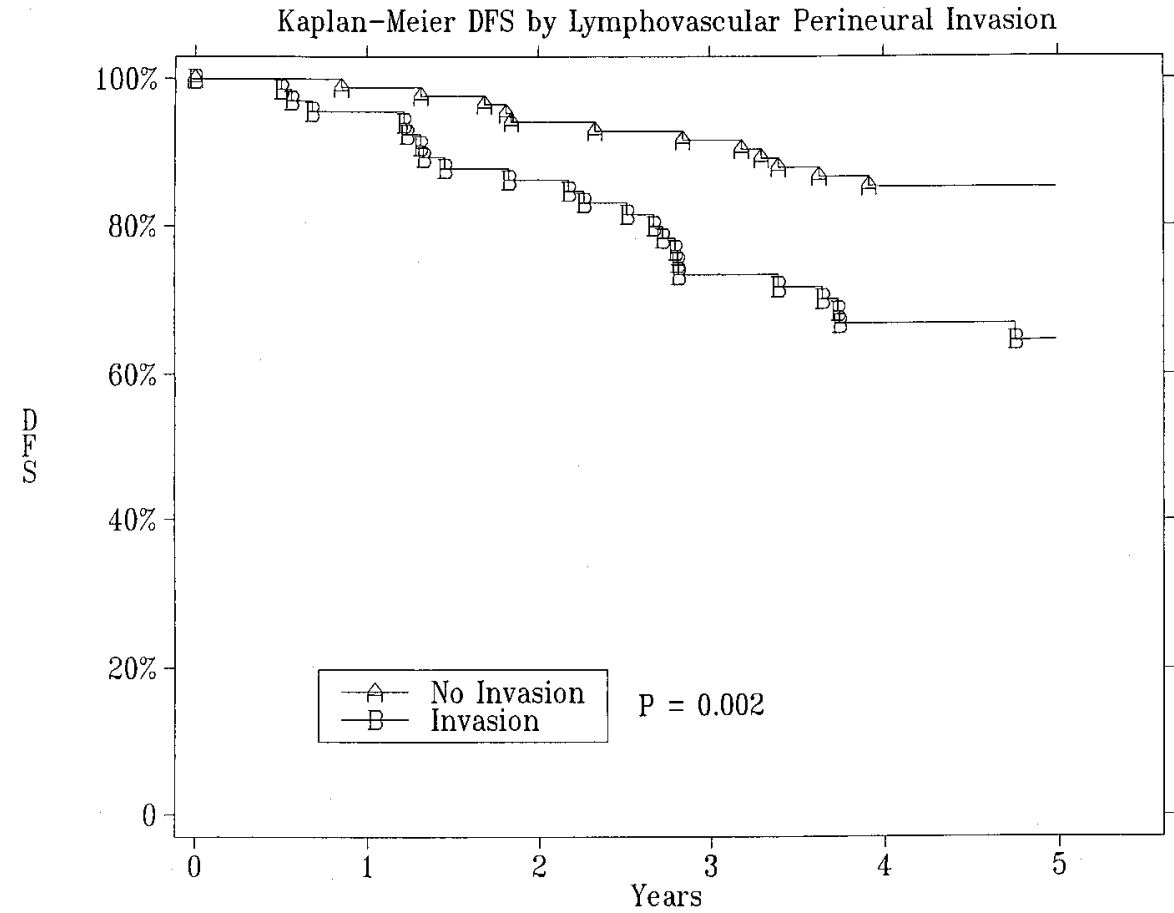

FIG. 7. Kaplan-Meier plot of distant DFS by LVPI (no invasion, invasion). The different types of invasion have been collapsed to simplify the plot, and the $P$ value changes accordingly from Table 1. 


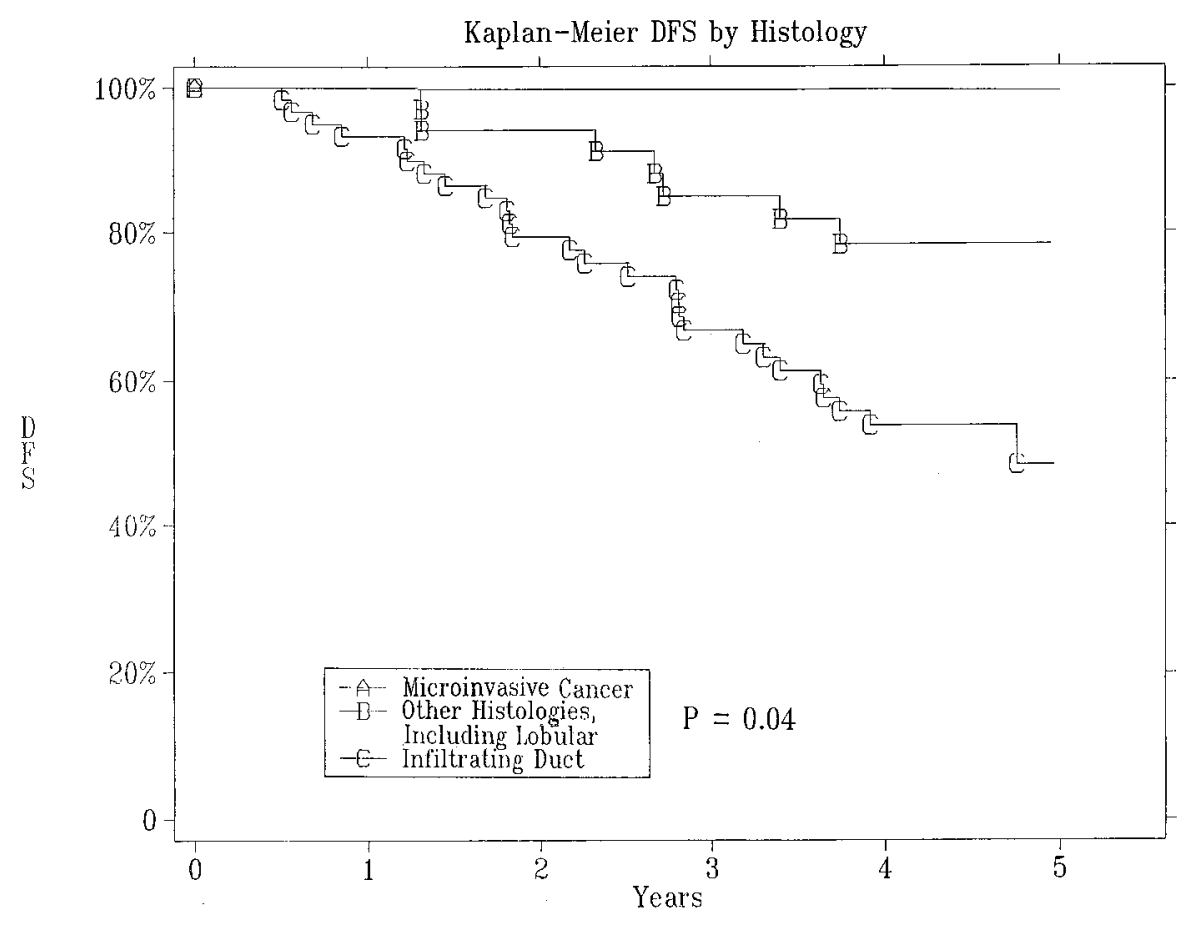

FIG. 8. Kaplan-Meier plot of distant DFS by histology (microinvasive cancer; other histologies, including lobular; infiltrating duct NOS). The category microinvasive cancer is referred to elsewhere in the article as predominantly DCIS; lobular carcinoma is grouped with other histologies for this plot; the $P$ value is unchanged from that in Table 1. benefit, even for node-negative patients, with the administration of adjuvant systemic therapy. However, our data reflect clinical practice; there was no uniform treatment protocol for the patients. Although the HBBC physicians made an effort to provide uniform treatment plans, patient preference certainly influenced treatment. We showed that there was a significant association $(P<.001)$ between the administration of adjuvant systemic therapy and nodal status, with $91 \%$ of node-positive patients and $31 \%$ of the node-negative patients receiving adjuvant systemic therapy. A lack of inclusion of systemic therapy in the step-wise model may just reflect a confounding with nodal status which was included.

In conclusion, we have demonstrated the importance of checking the appropriateness of the underlying assumption of proportional hazards, and of using an alternate model when there is strong evidence against this assumption. There was such evidence for nodal status; nodal status, one of the two factors considered to be of primary importance in predicting a patient's prognosis, was not included in the step-wise Cox model for DFS. Nodal status was included in the step-wise model with a log-normal model-type.

The effects of 20 prognostic factors (15 traditional, 5 newer prognostic factors) were considered in a multivariate context. The best step-wise model included only 5 traditional factors: tumor size, nodal status, nuclear grade, LVPI, and histology.
Acknowledgments: This work was supported by Physicians Services Incorporated, Women's College Hospital Research Fund, E.B. Fish Research Fund, and the Canadian Association of General Surgeons Research Fund.

We thank Dr. Betty G. Mobbs (retired) for the biochemical receptor assay data and Dr. John Blondal for Medical Oncology assessment. We also thank Jacqueline Wall, Gladys Oldfield, and Marilyn Link for their diligent assistance in data acquisition. The reviewers provided useful comments which strengthened the presentation of this work.

\section{REFERENCES}

1. Clark GM. Integrating prognostic factors. Breast Cancer Res Treat 1992;22:187-91.

2. Chapman JW, Murray D, McCready DR, et al. An improved statistical approach: can it clarify the role of new prognostic factors for breast cancer? Euro J Cancer 1996;32A:1949-56.

3. Chapman JW, Hanna W, Kahn HJ, Lickley HLA, Wall J, Fish EB, McCready DR. Alternative multivariate modelling for time to local recurrence for breast cancer patients receiving a lumpectomy alone. Surg Oncol 1996;5:265-71.

4. Chapman JW, Trudeau ME, Pritchard KI, et al. A comparison of all subset Cox and accelerated failure time models with Cox step-wise regression for node positive breast cancer. Breast Cancer Res Treat 1992;22:263-72.

5. Pritchard KI, Trudeau ME, Chapman JW, et al. Prognostic variables in node- negative breast cancer: an all subset analysis. Proc ASCO (Abstract) 1993;12:68.

6. Hilsenbeck SG, Ravdin PM, de Moor CA, Osborne CK, Clark GM Paradoxical decreases in prognostic utility as datasets mature: time-dependent lack of proportional hazards in prognostic factors in primary breast cancer. Breast Cancer Res Treat (Abstract) 1996;37:35. 
7. Boag JW. Maximum likelihood estimates of the proportion of patients cured by cancer therapy. JRSS B 1949;11:15-44.

8. Rutqvist LE, Wallgren A, Nilsson BO. Is breast cancer a curable disease? Cancer 1984;53:1793-800.

9. Gamel JW, Vogel RL, McLean IW. Assessing the impact of adjuvant therapy on cure rate for stage 2 breast carcinoma. $\mathrm{Br} \mathrm{J}$ Cancer 1993;68:115-8.

10. Gamel JW, Vogel RL. A model of long-term survival following adjuvant therapy for stage 2 breast cancer. Br J Cancer 1993;68: 1167-70.

11. Gamel JW, Vogel RL, Valaguisa P, Bonadonna G. Parametric survival analysis of adjuvant therapy for stage II breast cancer. Cancer 1994;74:2483-490.

12. Gamel JW, Meyer JS, Province MA. Proliferative rate by S-phase measurement may affect cure of breast carcinoma. Cancer 1995; 76:1009-18.

13. Berg JW. The distribution of cancer deaths in time. A survey test of the lognormal model. Br J Cancer 1965;19:695-711.

14. Kalbfleisch JD, Prentice RL. The Statistical Analysis of Failure Time Data. New York: John Wiley and Sons, 21-35, 1980.

15. Veronesi U, Marubini E, Del Vecchio M, et al. Local recurrences and distant metastases after conservative breast cancer treatments: partly independent events. J Natl Cancer Inst 1995;87:19-27.

16. Sun J, Chapman JW, Gordon R, Sivaramakrishna R, Link MA, Fish EB. Survival from primary breast cancer by tumour size for the age groups with different mammography screening guidelines. Breast Cancer Res Treat (Abstract) 1998;50:281.

17. Fan M, Chapman J, Link MA, Fish EB. Competing risks analysis for recurrence from primary breast cancer after lumpectomy. Breast Cancer Res Treat (Abstract) 1999;57:41.

18. Hallett D, Chapman JW, Gamel JW, McLean IW. Estimation of "cure" rate for intraocular melanoma. Proceed Am Assoc Cancer Res (Abstract)1999;40:43.
19. Chapman JW, Wolman E, Wolman SR, et al. Assessing genetic markers of tumour progression in the context of intra-tumour heterogeneity. Cytometry 1998;31:67-73.

20. Chapman JW, Mobbs BG, McCready DR, et al. An investigation of cut-points for primary breast cancer estrogen and progesterone receptor assays. J Steroid Biochem Mol Biol 1996;57:323-8.

21. Altman DG, Lausen B, Sauerbrei W, Schumacher M. Dangers of using "optimal" cutpoints in the evaluation of prognostic factors. J Natl Cancer Inst 1994;86:829-35.

22. Gore SM, Pocock SJ, Kerr GR. Regression models and nonproportional hazards in the analysis of breast cancer survival. Appl Statist 1984;33:176-95.

23. Allred DC, Harvey JM, Berardo M, Clark GM. Prognostic and predictive factors in breast cancer by immunohistochemical analysis. Mod Pathol 199811:155-68.

24. Hayes DF, Bast RC, Desch CE, et al. Tumor marker utility grading system: a framework to evaluate clinical utility of tumour markers. J Natl Cancer Inst 1996;88:1456-66.

25. Gusterson BA. The change in the role of the pathologist in the prediction of tumor behaviour and response to treatment. In: Dickson RB, Lippman HE eds. Doctors, Drugs and Hormonal Resistance in Breast Cancer. New York, Ellis Harwood, 1995, 39-53.

26. Locker AP, Ellis JO, Morgan DAL, Elston CW. Factors influencing local recurrence after excision and radiotherapy for primary breast cancer. Br J Surg 1989;76:890-4.

27. McCready DR, Hanna W, Kahn HJ, Chapman J, Wall J, Fish EB, Lickley HLA. Factors associated with local breast cancer recurrence after lumpectomy alone. Ann Surg Oncol 1996;3: $358-66$.

28. Andrulis IL, Bull SB, Blackstein ME, et al. Neu/erbB-2 amplification identifies a poor- prognosis group of women with nodenegative breast cancer. J Clin Oncol 1998;16:1340-9. 